ARTICLE

\title{
A recombined Sr26 and Sr61 disease resistance gene stack in wheat encodes unrelated NLR genes
}

Jianping Zhang (1) 1,2, Timothy C. Hewitt (10 1,2, Willem H. P. Boshoff ${ }^{3}$, lan Dundas ${ }^{4}$, Narayana Upadhyaya (D) ${ }^{2}$, Jianbo Li ${ }^{1}$, Mehran Patpour (1) ${ }^{5}$, Sutha Chandramohan², Zacharias A. Pretorius (1) ${ }^{3}$, Mogens Hovmøller ${ }^{5}$, Wendelin Schnippenkoetter (10 2, Robert F. Park (10 1, Rohit Mago (1) 2, Sambasivam Periyannan², Dhara Bhatt ${ }^{2}$, Sami Hoxha', Soma Chakraborty², Ming Luo ${ }^{2}$, Peter Dodds (1) ${ }^{2}$, Burkhard Steuernagel (10 ${ }^{6}$, Brande B. H. Wulff ${ }^{6}$, Michael Ayliffe ${ }^{2}$, Robert A. Mclntosh ${ }^{1}$, Peng Zhang (1) ${ }^{1 凶}$ \& Evans S. Lagudah (1) ${ }^{1,2 凶}$

The re-emergence of stem rust on wheat in Europe and Africa is reinforcing the ongoing need for durable resistance gene deployment. Here, we isolate from wheat, Sr26 and Sr61, with both genes independently introduced as alien chromosome introgressions from tall wheat grass (Thinopyrum ponticum). Mutational genomics and targeted exome capture identify Sr26 and Sr61 as separate single genes that encode unrelated (34.8\%) nucleotide binding site leucine rich repeat proteins. Sr26 and Sr61 are each validated by transgenic complementation using endogenous and/or heterologous promoter sequences. Sr61 orthologs are absent from current Thinopyrum elongatum and wheat pan genome sequences, contrasting with Sr26 where homologues are present. Using gene-specific markers, we validate the presence of both genes on a single recombinant alien segment developed in wheat. The co-location of these genes on a small non-recombinogenic segment simplifies their deployment as a gene stack and potentially enhances their resistance durability.

\footnotetext{
${ }^{1}$ Plant Breeding Institute, School of Life and Environmental Sciences, University of Sydney, Cobbitty, NSW, Australia. ${ }^{2}$ CSIRO Agriculture \& Food, Canberra, ACT, Australia. ${ }^{3}$ Department of Plant Sciences, University of the Free State, Bloemfontein, South Africa. ${ }^{4}$ School of Agriculture, Food and Wine, University of Adelaide, Urrbrae, SA, Australia. ${ }^{5}$ Department of Agroecology, Aarhus University, Slagelse, Denmark. ${ }^{6}$ John Innes Centre, Norwich Research Park,

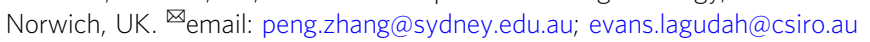


$\mathrm{T}$ he emergence of widely virulent Puccinia graminis f. sp. tritici (Pgt) races over the past two decades ${ }^{1,2}$ has motivated global efforts to identify effective stem rust (Sr) resistance genes. During the last seven years, nine seedling (or all stage) Sr genes (viz. Sr13, Sr21, Sr22, Sr33, Sr35, Sr45, Sr46, Sr50, and $S r 60$ ) have been cloned, eight of which encode nucleotidebinding, leucine-rich-repeat (NLR) immune receptors ${ }^{3-9}$. Sr60 is an exception that encodes a tandem kinase protein ${ }^{10}$. These genes were targeted due to their effectiveness against Ug99 and other Pgt races. Their sequences now provide perfect markers and diagnostic tools for marker-assisted breeding. However, the subsequent appearance of new, diverse virulent isolates means that most of these cloned $S r$ genes have been overcome by at least one Pgt race within and/or beyond the Ug99 lineage. Consequently, there is an ongoing need to expand resistance resources and to enhance gene stewardship through co-deployment of multiple resitance $(R)$ genes, rather than single genes, to increase resistance durability.

Grass species related to wheat carry sources of resistance that can be transferred to wheat. $\mathrm{Sr} 26$ is derived from tall wheat grass [Thinopyrum ponticum $(2 n=10 \times=70)$ ], and its introgression into common wheat as a T6AS.6AL-6Ae\#1 translocation chromosome is an early example of a transfer of resistance from a wheat wild relative ${ }^{1,12}$. Sr26 was transferred to wheat chromosome $6 \mathrm{~A}$ by seed irradiation in the early 1960s and this resistance has remained effective against all tested Pgt races, including those in the Ug99 group ${ }^{11,13-15}$. A second Th. ponticum-derived $\mathrm{Sr}$ gene, Sr61 (previously designated $\mathrm{SrB}$ ), was identified in South African wheat accession W3757, which carries a 6Ae\#3 (6D) chromosome substitution ${ }^{16}$. No Pgt virulence has been reported for Sr61 either. Sr26 has been deployed in a number of Australian wheat cultivars since 1971 and has likely fulfilled the definition of durable resistance ${ }^{17}$. Sr61 on the other hand has not been deployed in a cultivar. Since genetic dissection of genes within alien segments in wheat was not possible due to lack of recombination, the question arose as to whether the apparent durability of such resistances might be due to multiple genes rather than a single gene. Resistance in W3757 was located on chromosome 6Ae\#3, making it possible that $\operatorname{Sr} 26$ and $\operatorname{Sr} 61$ are alleles or related paralogues ${ }^{18}$.

It is noteworthy that neither of these Th. ponticum segments can recombine with wheat chromatin; however, they can recombine with each other when both are present in wheat. Recently, a recombinant line was developed in which Sr61 was transferred from 6Ae\#3 to a T6AS.6AL-6Ae\#1 translocation segment by homologous recombination ${ }^{19}$ with the 6Ae\#1 segment carrying Sr26. However, as there is no current Pgt race known to be virulent to either resistance gene, it was not possible to unambiguously determine whether the recombinant introgression carried a single or both genes. Molecular markers developed for Sr26 and Sr61 were not reliable indicators of the Sr genes per se, as they were based on DNA sequences that were located at unknown positions on the entire non-recombinogenic alien segments or alternatively, present in large linkage blocks of unordered markers that cosegregated with the resistance ${ }^{19-22}$. The ambiguity surrounding the presence of both genes in the potential recombinant segment was therefore difficult to resolve using traditional methods. After more than 40 years following their characterization in the 1980s we have used cloning to determine the relationship existing between these two very useful $R$ genes.

Here, we show that $S r 26$ and $S r 61$ encode unrelated NLR genes that have been combined in a single Th. ponticum segment representing a natural gene stack, that simplifies codeployment of these two resistance genes and potentially enhances their resistance durability.

\section{Results}

Identification of a Sr26 candidate gene by MutRenSeq. Conventional map-based cloning of $S r 26$ and $S r 61$ in wheat derivatives was not feasible due to the absence of recombination between common wheat and alien chromosome segments. Target-sequence Enrichment and Sequencing (TEnSeq) pipelines developed in recent years have overcome such constraints to map-based cloning ${ }^{23}$. Here we used mutational genomics and targeted exome capture of NLR immune receptor genes, a method termed MutRenSeq ${ }^{8}$, for isolation of Sr26 and Sr61. For Sr26 cloning we screened $1270 \mathrm{M}_{2}$ lines and identified five susceptible ethyl methanesulfonate (EMS)-induced mutants from the Sr26carrying wheat genetic stock, Avocet $+\operatorname{Lr} 46^{22}$ (Fig. 1a and Supplementary Table 1). One mutant (150S) carried a deletion of linked marker $\# 43^{20}$. Because there was no information regarding the genetic distance from the linked marker, we hypothesized that simultaneous loss of a marker with loss of the resistance was indicative of a deletion of unknown size ${ }^{20}$. The five mutants, together with wild-type Avocet+Lr46, were subjected to NLR gene exome capture, sequencing and alignment using MutantHunter ${ }^{8}$ (Supplementary Fig. 1a). A single contig of 2466 bp that was absent from the putative deletion mutant $150 \mathrm{~S}$ contained a single nonsynonymous nucleotide change in each of the remaining four mutants. The full length gene isolated from Avocet+Lr46 encodes a 935 amino acid (aa) protein consisting of a coiled-coil (CC) domain at the N-terminus, a NB-ARC domain and LRR motifs at the C-terminus (CNL) (Fig. 1b). Three mutants encoded amino acid changes in conserved motifs in the NB-ARC domain; Ala311Thr (RNBS-C motif) in mutant 128S and a common Ser431Asn (RNBS-D motif) mutation in mutants $70 \mathrm{~S}$ and $12 \mathrm{~S}$, suggesting the latter two mutants were sibs that inherited the same mutation event. The nucleotide change in mutant 499 S was a premature stop codon (Supplementary Fig. 2).

Transgenic validation of the Sr26 candidate gene. A transgenic complementation experiment was undertaken to confirm the function of the $S r 26$ gene candiate in wheat cultivar Fielder. The assembled genomic sequence available for the Sr26 candidate included $917 \mathrm{bp} 5^{\prime}$ of the translational start codon and $263 \mathrm{bp} 3^{\prime}$ of the stop codon, and therefore may lack sufficient regulatory sequences for appropriate gene expression (Fig. 2a). To ensure candidate gene expression, three constructs were used to produce transgenic plants (Fig. 2a). One construct comprising the available native sequences described above was designated Sr26:NativeRE (Regulatory Elements). The other two, designated Sr26: Sr22RE and Sr26:Sr33RE, combined the Sr26:NativeRE construct with $5^{\prime}$ and $3^{\prime}$ regulatory elements derived from wheat stem rust resistance genes $\mathrm{Sr} 22$ and $\mathrm{Sr} 33$, respectively ${ }^{7,8}$ (Fig. 2a). A previous report had shown that $\mathrm{Sr} 45$ gene function was retained when expressed using $S r 33$ regulatory elements ${ }^{24}$, suggesting these heterologous regulatory sequences could be used for $\mathrm{Sr} 26$ expression.

We generated 21,22, and 14 independent primary transgenic Fielder lines carrying the Sr26:NativeRE, Sr26:Sr22RE, and Sr26: Sr33RE constructs, respectively. All 57 independent $T_{0}$ plants were resistant to a $P g t$ isolate of race $98-1,2,3,5,6+\operatorname{Sr} 50$, whereas all non-transformed Fielder controls were susceptible (Fig. $2 \mathrm{~b}$ and Supplementary Table 2). These data indicated that the minimal endogenous promoter and terminator sequences used for the Sr26:NativeRE construct were sufficient for functional $\mathrm{Sr} 26$ expression, as was the addition of $\mathrm{Sr} 22$ and $\mathrm{Sr} 33$ promoter and terminator sequences. To confirm the resistance cosegregated with the transgene, two $T_{1}$ families of 15 and 16 progeny from line PC235-5 and PC235-4 containing the Sr26:NativeRE transgene were analysed (Supplementary Fig. 3a, b). Transgene 
a

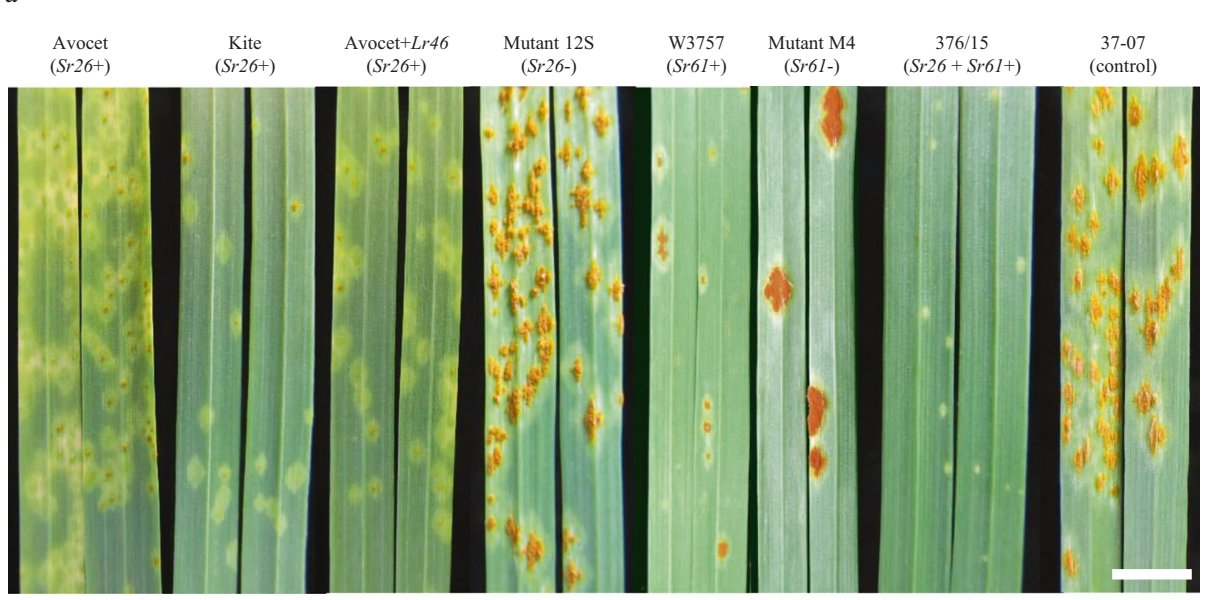

b
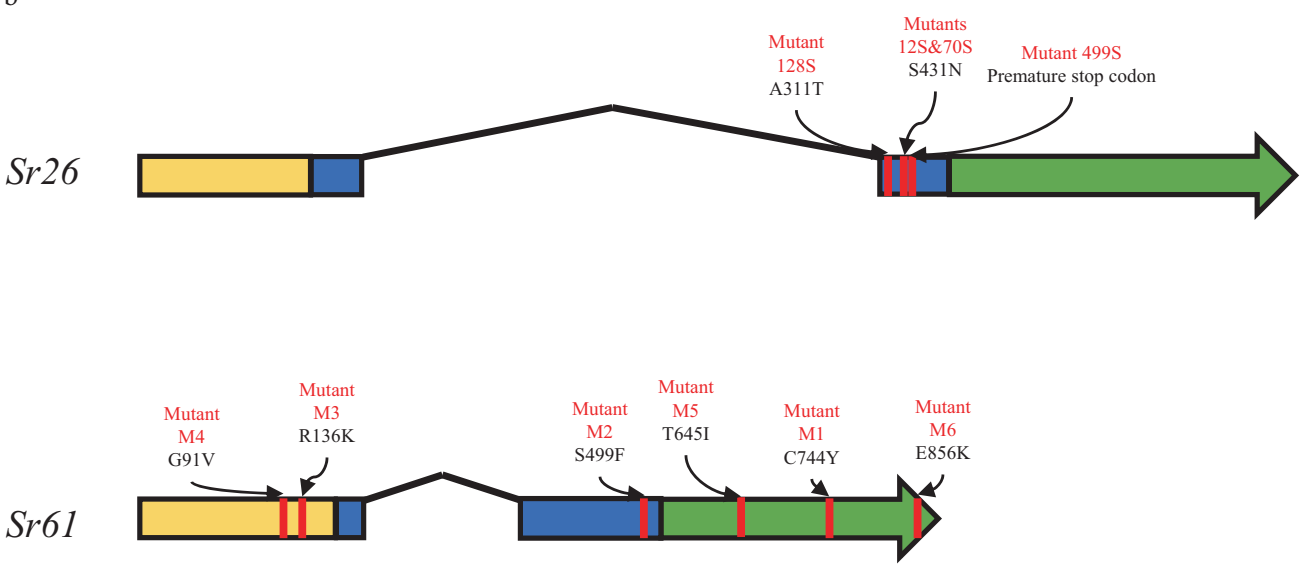

Fig. 1 Rust response phenotypes conferred by the Sr26 and Sr61 parental sources and mutant derivatives, and gene structures of Sr26 and Sr61 candidate genes. a Abaxial seedling leaf surfaces of wild-type and representative EMS-generated susceptible mutants for Sr26 and Sr61, together with recombinant 376/15 inoculated with Pgt race PTKST. Avocet, Kite, Avocet+Lr46, W3757, and recombinant 376/15 showed low infection types (small pustules or flecking), whereas Sr26 mutant 12S, Sr61 mutant M4 and susceptible control line 37-07 all showed high infection types (large pustules). Bar shows $1 \mathrm{~cm}$. $\mathbf{b}$ Gene structures of candidate Sr26 and Sr61 genes. Exons are shown as solid boxes with the terminal exon indicated as an arrow to show the direction of transcription whereas introns are shown as solid black angled lines. The position of mutations in mutant lines are shown in red with predicted amino acid substitutions caused by nonsense mutations and premature stop codon shown above. CC domain, NB domain, and LRR motifs are shaded upon the exons with yellow, blue, and green colors.

copy number was determined in PC235-5 by quantitative PCR and a minimum of two transgene loci, that segregated independently were detected based upon gene copy number (Supplementary Fig. 3c). All transgenics with at least one transgene copy (i.e., hemizygous) were resistant whereas the null segregant and Fielder control were susceptible (Supplementary Fig. 3c). Forty-six $T_{1}$ plants derived from four independent transgenic events, two Sr26:Sr22RE and two Sr26:Sr33RE events, were all resistant to $P g t$ race $98-1,2,3,5,6+$ Sr50 whereas all Fielder controls lacking the transgene were susceptible (Supplementary Fig. 3d, e). These latter heterologous regulatory sequences caused no change in the Sr26 resistance phenotype seen in transgenic plants (Supplementary Fig. 3a, b, d, e).

Identification of an Sr61 candidate gene by MutRenSeq. We identified eight susceptible EMS mutants derived from line W3757 among $1837 \mathrm{M}_{2}$ lines screened. Two mutants contained deletions of a previously reported marker, MWG798, linked to $S r 61^{19}$. The remaining six mutants (M1-M6) were potential point mutations and together with wild-type W3757 were analysed by NLR gene capture and sequencing (Fig. 1a and Supplementary
Fig. 1b). A single contig was identified that contained nucleotide changes in five of the six mutants (M1-M5) (Supplementary Fig. 1b). The full length gene of 3519 bp isolated from W3757 encodes an 880 aa protein containing a coiled-coil (CC) domain, NB-ARC domain and LRR motifs (Fig. 1b). A nucleotide substitution was subsequently identified in the sixth mutant (M6) in the sequence present in the full-length gene that was absent in the original MutRenSeq contig. Each mutant contained nonsynonymous nucleotide changes that caused amino acid substitutions in either the CC (M3, M4), NB-ARC (M2), or LRR (M1, M5, and M6) domains (Fig. $1 \mathrm{~b}$ and Supplementary Fig. 2).

Transgenic validation of the Sr61 candidate gene. Transgenic complementation in wheat cultivar Fielder was used to confirm the function of the Sr61 gene candidate. The assembled genomic sequence available for the Sr61 candidate encoded only 354 bp $5^{\prime}$ and $67 \mathrm{bp} 3^{\prime}$ of the gene translation start and stop codons, respectively. To ensure the expression of the candidate gene a heterologous construct designated Sr61:Sr26RE was produced encoding the Sr61 genomic sequence, and the $5^{\prime}$ and $3^{\prime}$ Sr26 regulatory elements shown above to function in the Sr26:NativeRE 
a

Fielder:Sr26:NativeRE

Fielder:Sr26:Sr22RE

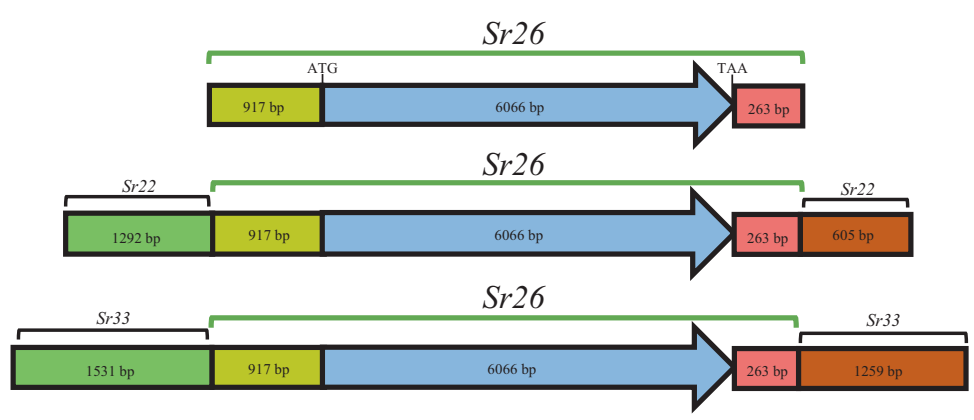

Fielder:Sr61:Sr26RE

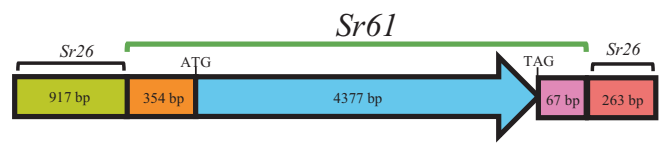

$\mathrm{b}$

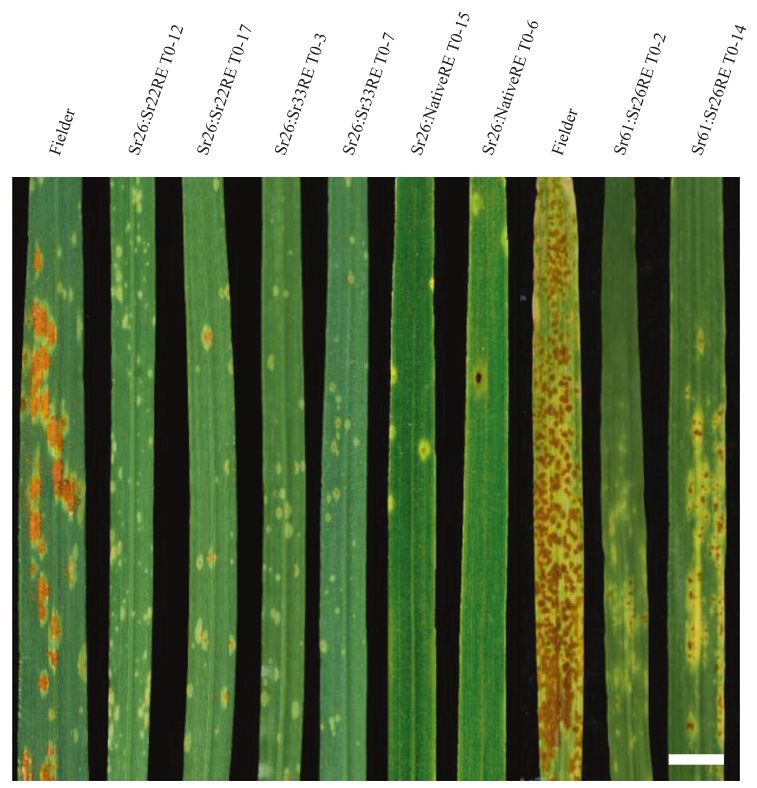

Fig. 2 Validation of the Sr26 and Sr61 candidate genes by transformation. a Four constructs used for wheat transformation. The three Sr26 constructs encoded the Sr26 gene candidate and either its native regulatory sequences (Sr26:NativeRE), regulatory sequences from Sr22 (Sr26:Sr22RE) or regulatory sequences from Sr33 (Sr26:Sr33RE). A single construct was used for the Sr61 gene candidate under the regulatory control of Sr26 5' and 3' regulatory elements (REs). 5' REs and 3' REs are indicated by black brackets with sizes in bp indicated for all four constructs. Sr26 and Sr61 intron/exon coding regions are shown as light and dark blue arrows. b Phenotypic responses of representative $T_{0}$ plants produced for all four constructs and inoculated with Pgt race $98-1,2,3,5,6+$ Sr50 along with non-transgenic Fielder control lines. All lines except the susceptible Fielder control showed low infection types. Photographed 12 days of post-inoculation. Bar shows $1 \mathrm{~cm}$.

construct (Fig. 2a). We generated 21 independent primary transgenic lines carrying the Sr61:Sr26RE construct. From these 21 lines, $14 \mathrm{~T}_{0}$ plants at a similar growth stage were selected and inoculated with the Pgt race 98-1,2,3,5,6+Sr50 isolate. All 14 lines were resistant whereas non-transformed Fielder controls were susceptible (Fig. 2b and Supplementary Table 2). Ten transgenic plants from three independent $\mathrm{T}_{1}$ families were chosen to compare $\mathrm{Pgt}$ resistance phenotypes with transgene copy number and expression (Supplementary Fig. 3f). Eight plants contained at least one transgene copy and all were resistant except two plants, Sr61: Sr26RE $\mathrm{T}_{1}-6-7$ and Sr61:Sr26RE $\mathrm{T}_{1}$-17-11, which had a high infection type. qRT-PCR was then performed on all $10 \mathrm{~T}_{1}$ lines and the results revealed that plant Sr61:Sr26RE $T_{1}-17-11$ had a single transgene copy that had very low expression, when compared with other transgene positive plants (Supplementary Fig. 3f). A resistant sib of this plant $\left(\mathrm{T}_{1}-17-2\right)$ contained two additional transgene copies suggesting that at least two independent transgene loci segregated in this family, one of which had sufficient expression for Sr61 resistance (Supplementary Fig. 3f). Similarly, plant Sr61: Sr26RE $T_{1}-6-7$ showed no detectable Sr61 transcript expression, despite an apparently high transgene copy number, whereas its sibling line Sr61:Sr26RE $\mathrm{T}_{1}-6-3$ expressed the transgene normally, again suggesting the segregation of independent expressing and non-expressing transgenic loci in this line (Supplementary Fig. 3f).

Application of Sr26 and Sr61 in developing wheat breeding germplasm. To facilitate the use of Sr26 and Sr61 in breeding and permit their reliable identification in the recombinant introgression segment described previously ${ }^{19}$, we developed gene-specific markers for each gene. For Sr26, a 1580 bp PCR amplicon was identified that flanked the intron I-exon II junction and was highly specific for Sr26 (Supplementary Fig. 4a). For Sr61, a marker with an amplicon size of 278 bp located in the first exon was confirmed to be Sr61-specific (Supplementary Fig. $4 \mathrm{~b}$ and 
Supplementary Table 3). Using these markers, we confirmed the presence of both Sr26 and Sr61 in the recombinant line (Supplementary Fig. 4). Molecular cytogenetic analysis showed that the alien segment in the recombinant line was substantially smaller than that in both the T6AS.6AL-6Ae\#1 translocation ${ }^{22}$ and $6 \mathrm{Ae} \# 3$ chromosome substitution lines (Supplementary Fig. 5).

Sr26 and Sr61 homologs in the Thinopyrum and Chinese Spring genomes. We searched for homologs of Sr26 and Sr61 in the recently available $T h$. elongatum genome sequence ${ }^{25}$ and the Chinese Spring genome. Sequences with greatest homology to Sr26 coding sequences was located on the distal end of the group 6 chromosome of the E subgenome, and on the distal end of chromosome 6B in Chinese Spring. This is consistent with high synteny existing between the group 6 chromosomes of wheat $(6 \mathrm{~A}$, $6 \mathrm{~B}$, and 6D) and chromosome 6E of Th. elongatum (Supplementary Table 4), and the location of Sr26 in the T6AS.6AL$6 \mathrm{Ae} \# 1$ translocation. However, the best hits of $\mathrm{Sr} 61$ were located on the proximal region of $6 \mathrm{E}$ in Th. elongatum or on $2 \mathrm{D}$ or $7 \mathrm{D}$ in Chinese Spring and showed poor matches with less than 61 and $69 \%$ of the gene covered. This suggests that these genomes do not include orthologous genes to Sr61.

Phylogenetic analysis of Sr26 and Sr61. To determine the evolutionary relationship of Sr26 and Sr61 to other plant known R proteins of the CNL class, we generated a phylogenetic tree based on the alignment of 123 proteins and used the L6 flax rust resistance Toll/interleukin-1 receptor (TIR) NLR protein as an outgroup (Fig. 3a and Supplementary Data 1) ${ }^{26}$. Three large clades (Clade I-III) and a small clade (Clade IV) were identified (Fig. 3a). Although both Sr26 and Sr61 originated from tall wheat grass, the most closely related R protein to Sr26 was encoded by the Triticum turgidum ssp. dicoccum Sr 13 gene (58.46\% identity at protein level) (Fig. 3b). The Sr61 protein is less similar to both
Sr13 (35.21\%) and Sr26 (34.81\%), but all three proteins are members of a clade that includes Sr22, Sr33, Sr35, Sr50, Sr46, and proteins encoded by the barley Mla $R$ gene family (Fig. 3a, Clade I). By contrast $\mathrm{Sr} 21$ and $\mathrm{Sr} 45$ are in a clade more closely related to wheat powdery mildew $\mathrm{R}$ proteins encoded by $\mathrm{Pm} 3$ alleles (Fig. 3a, Clade II).

Resistance spectra of Sr26 and Sr61. The resistance responses conferred by $S r 26$ and Sr61 were tested against six and eight Pgt isolates, respectively, including races PTKST (collected in South Africa), TTRTF (collected in Italy and Eritrea), and TTKTT (collected in Kenya) (Supplementary Fig. 6 and Supplementary Tables 5 and 6). Screening of wild-type and mutant lines demonstrated that Sr26 unequivocally provided resistance to all six isolates, whereas $\mathrm{Sr} 61$ gave resistance to at least five of eight isolates. Additional resistance genes in the background of line W3757 were epistatic to Sr61 masking its effectiveness against the other three isolates. In all assays, the three recombinant plants containing both gene candidates were consistently more resistant than lines with only one respective gene (Fig. 1 and Supplementary Figs. 6 and 7).

\section{Discussion}

Among the many wild relatives of $T$. aestivum, perennial wheat grasses (polyploid Thinopyrum spp.) are a recognized source of desirable traits for tolerance of wheat to both biotic and abiotic stresses, such as diseases, pests, salinity, and drought ${ }^{27}$. Remarkably, six stem rust resistance genes (Sr24, Sr25, Sr26, Sr43, Sr44, and Sr61), four leaf rust resistance genes ( $\operatorname{Lr} 19, \operatorname{Lr} 24, \operatorname{Lr} 29$, Lr38), two powdery mildew resistance genes (Pm40, Pm43), two barley yellow dwarf virus resistance genes $(B d v 2, B d v 3)$, and a recently cloned wheat Fusarium head blight resistance gene (Fhb7) were transferred from $T h$. spp. to wheat as summarized by Wang et al. ${ }^{25}$. The recently published diploid Th. elongatum genome assembly will further facilitate the transfer of genetic

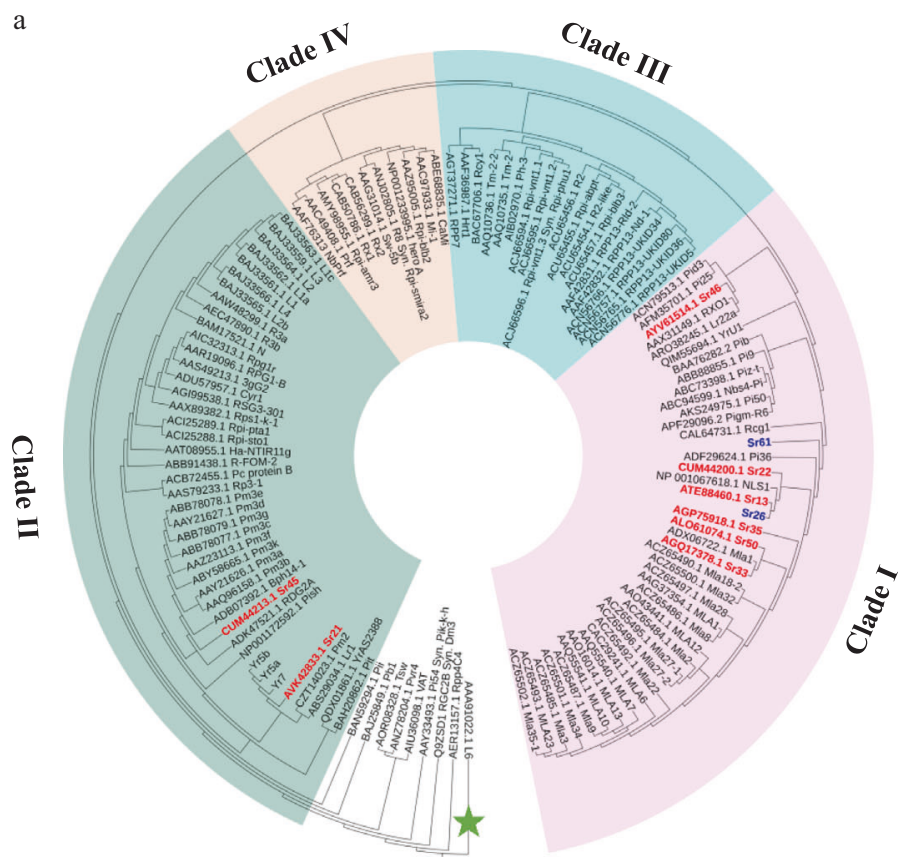

b

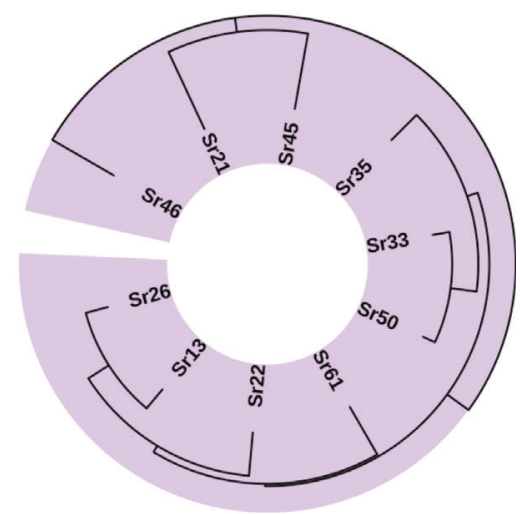

Fig. 3 Phylogenetic relationship between Sr26, Sr61 and other CC-NLR immune receptors from plants. a Comparison of 123 NLR type proteins formed three large clades (I-III) and one smaller clade (IV). The TIR type NLR protein encoded by the Linum usitatissimum L6 rust resistance gene was used as an outgroup (green star). Previously cloned wheat stem rust genes are in red script; Sr26 and Sr61 are in blue; and Clades I, II, III, IV are shaded in purple, green, blue, and light orange, respectively. b Simplified phylogenetic tree showing the evolutionary relationship between all ten cloned wheat stem rust $R$ genes that encode CC-NLR proteins. 
resources from Thinpopyrum spp. into cultivated wheat ${ }^{25}$. Here, we report the characterization of two such Th. ponticum stem rust resistance genes, Sr26 and Sr61, and show that they encode unrelated NLR proteins.

Using cytogenetic analysis we identified a close to entire chromosome arm 6Ae\#1 present in Avocet $+\mathrm{Lr} 46$ and a complete chromosome 6Ae\#3 in W3757 (Supplementary Fig. 5). These large chromosomal segments raised the possibility that the Sr26 and Sr61 resistance phenotypes could each be conferred by more than a single resistance gene carried within the large non-recombinogenic region. For example, Sr32 resistance was first identifed as conferred by a large introgressed segment of Aegilops speltoides chromosome 2S\#1 introduced by E.R. Sears ${ }^{28}$. Subsequent reduction of this segment by homoeologous recombination revealed that two different regions of this segment contain functional resistance genes, one of which was designated as Sr32 and the second as SrAest1t, indicating that the orginal introgression carried at least two resistance genes ${ }^{29}$. Similarly, three different stem rust resistance genes, Sr39, SrAest7t, and $\mathrm{Sr} 47$, were also identifed in translocation involving a $2 \mathrm{~S}$ chromosome (2S\#2) derived from a different Ae. speltoides accession ${ }^{29}$. However, for each of the Th. ponticum segments, all mutants susceptible to Pgt race 34-1,2,3,4,5,6,7 contained deficiencies in a single NLR gene, confirming that a single gene in each segment confers resistance to this isolate. Screening of these mutants with geographically diverse $P g t$ races showed that all six $P g t$ races screened were virulent on the Sr26- Avocet+Lr46 mutants, suggesting that there is likely only one resistance gene on the original Sr26 introgression segment. In contrast, 5 out of $8 \mathrm{Pgt}$ races were virulent on W3757 mutants, indicating the presence a single gene effective against these isolates. However, additional resistance in W3757 masked the phenoype of Sr61 in tests with three Pgt isolates. It is unknown if this additional resistance is also associated with the Th. ponticum introgression. Previously Singh and McIntosh postulated the presence of Sr6 (located on chromosome 2D) and Sr12 (chromosome 3B) in addition to Sr61 in W3757 ${ }^{16}$. The three Pgt isolates (races TTKTF, TTKTT, and TTRTF) that were avirulent to W3757 mutants (Supplementary Table 5) are each virulent to Sr6 precluding this gene as providing background resistance whereas their pathogenicity to $\operatorname{Sr} 12$ was uncharacterized ${ }^{28}$. Nevertheless, given its unusual requirment of lower tempearture for incubation period, Sr12 also seems unlikely to provide seedling resistance in any of our disease resistance tests ${ }^{28}$. It remains possible that additional unknown resistance genes could be present in W3757 either on the introgressed segment or elsewhere in the genome. Any other resistances encoded on these introgressions provide limited resistance when compared with the two genes we have isolated, thus, we have designated these genes Sr26 and Sr61, respectively.

Due to only short $5^{\prime}$ and $3^{\prime}$ noncoding sequences being available from both Sr26 and particularly Sr61, we used heterologous regulatory sequences from $\mathrm{Sr} 22$ and $\mathrm{Sr} 33$ for transgene expression. Luo et al. ${ }^{30}$ recently showed substantial variation existed between the expression levels of a number of different NLR encoding $S r$ genes, with the $S r 50$ gene expressed at an approximately 10-fold higher level when compared with Sr22. Using Sr26 regulatory elements we saw an approximately $40 \%$ reduction in Sr61 transgene expression in resistant transgenics compared with the endogenous gene present in W3757; however, the resistance phenotype appeared unchanged. Similarly Sr22 and Sr33 regulatory sequences were sufficient to express $S r 26$ and provided levels of resistance similar to the Sr26 transgene containing native, albeit short, regulatory sequences and the endogenous Sr26 gene in Avocet.

The tertiary wheat genepool genes, Sr26 and Sr61, encode NLR proteins, like eight of the nine previously cloned wheat $\mathrm{Sr}$ genes $^{3-9}$. Amongst cloned wheat stripe rust all-stage resistance genes, the $\operatorname{Yr} 15$ gene encodes a tandem kinase domain protein and $Y r 5 a, Y r 5 b$, and $Y r 7$ encode NLR proteins with integrated BED domain motifs ${ }^{31,32}$. In contrast, $Y r U 1$ and $Y r A S 2388$ encode canonical NLR proteins ${ }^{33,34}$. A number of NLR proteins are also the products of wheat leaf rust all-stage resistance genes (i.e., $\operatorname{Lr} 1$, Lr21) while $\operatorname{Lr} 10$ requires dual NLR proteins for function ${ }^{35-37}$. Therefore, NLR proteins predominate in providing all-stage resistance to all three fungal rust species in wheat and its immediate ancestors. However, other proteins can also contribute, such as the recently reported membrane bound ankyrin repeat protein encoded by $\operatorname{Lr} 14 a^{38}$.

Combining multiple $R$ genes is a widely accepted gene stewardship strategy to enhance the durability of resistance on the basis that the simultaneous defeat of two or more effective $R$ genes is less likely than defeat of either gene alone. One compelling reason for cloning $R$ genes is the opportunity to combine them into transgenic cassettes to allow multiple $R$ genes to be selected as a single "trait" in breeding ${ }^{30}$. The most desirable $\mathrm{Sr}$ genes to be combined into a transgene cassette are those with broad effectiveness against diverse Pgt races. Virulence to most of the currently cloned $\mathrm{Sr}$ genes in wheat has been documented making isolation of $\operatorname{Sr} 26$ and $S r 61$ a valuable addition for inclusion into future transgenic cassettes.

However, transgene cassette deployment in wheat is currently constrained by GM regulations ${ }^{39}$. Here, we confirm the presence of both $\mathrm{Sr} 26$ and $\mathrm{Sr} 61$ in a recombinant alien introgression segment obtained by homologous recombination. This alien segment derived from tall wheat grass does not recombine with wheat chromosomes making it a combined Sr26-Sr61 gene stack. In addition, this alien segment is considerably shorter than that in Sr26-bearing lines that have been used in commercial wheat cultivars, decreasing the likelihood of unwanted linkage drag. This alien gene stack containing both Sr26 and Sr61 offers a nontransgenic route for co-inheritance of both $R$ genes and potentially increases the durability of both valuable resistance genes. However, that will occur only if the same genes are not exploited singly. Sr26 is already deployed alone, but Sr61 is currently present only in the presently reported lines.

\section{Methods}

Plant materials, mutagenesis, and mutant DNA preparation. Wheat lines carrying Sr26 (Avocet+Lr46) and Sr61 (W3757) were mutagenised with EMS and progeny susceptible to $P g t$ race $34-1,2,3,4,5,6,7$ were selected as described in a related study ${ }^{22}$. Genomic DNA was prepared from seedling leaves as described by Yu et al. ${ }^{40}$. DNA quality and quantity were assessed with a NanoDrop spectrophotometer (Thermo Fisher Scientific, Waltham, MA, USA) and by electrophoresis in $0.8 \%$ agarose gels.

R gene enrichment and sequencing. Target sequence enrichment of NLRs was undertaken by Arbor Biosciences (Ann Arbor, MI, USA) using the MYbaits protocol and the Triticeae NLR bait libraries Tv2 for Sr26 and Tv3 for Sr61, available at https:// github.com/steuernb/MutantHunter/blob/master/Triticea_RenSeq_Baits_V3.fasta.gz 8 . Library construction was undertaken using the TruSeq RNA Protocol v2. All enriched libraries were sequenced using a HiSeq 2500 (Illumina) sequencing platform that generated 250 bp paired-end reads. Sequencing data of wild-type and Sr26 and Sr61 mutants are summarized in Supplementary Data 2.

Sequence analysis. CLC Genomics Workbench v9.0 (Sr26) and v10.0 (Sr61) (Qiagen, Hilden, Germany) were used for read quality control, trimming, and de novo assembly of wild-type reads using the following parameters; minimum contig length: 250, auto-detect paired distances, perform scaffolding, mismatch cost: 2 , insertion cost: 3 , deletion cost: 3 , length fraction from 0.5 to 0.9 , and similarity fraction $0.9-0.98$. For mapping sequence reads from wild-type and mutants against the de novo wild-type assembly the following parameters were used; no masking, linear gap cost, length fraction $0.5-0.9$, and similarity fraction $0.95-0.98$. Sr26 contigs containing mutations in each line were identified using the MutantHunter ${ }^{8}$ pipeline with default parameters whereas the MuTrigo Python package (https:// github.com/TC-Hewitt/MuTrigo) was used for SNP calling with default parameters to identify candidate contigs containing mutations in the Sr61 mutants. For Sr61 analysis the mutant M1 sequence was used for de novo assembly due to insufficient data obtained from the wild-type (W3757) (Supplementary Data 2). 
Gene sequence confirmation. Total RNA was extracted using a PureLink ${ }^{\mathrm{TM}} \mathrm{RNA}$ Mini Kit (Invitrogen, Carlsbad, CA, USA) as per the manufacturer's instructions. cDNA synthesis was performed as described by Clontech (Mountain View, CA, USA). Flanking gene sequences were first amplified by $5^{\prime}$ and $3^{\prime}$ RACE (rapid amplification of cDNA ends) and then by using a Universal GenomeWalker kit (Takara Bio, Mountain View, CA, USA). Nonsynonymous substitutions identified in mutants by RenSeq were confirmed by PCR amplification of mutant DNAs and Sanger sequencing (Supplementary Table 3). Exon-intron structures were confirmed by cDNA amplification and sequencing.

Candidate gene confirmation by wheat transformation. Sr26 and Sr61 gene candidates were introduced into wheat cultivar Fielder by Agrobacterium-mediated transformation using binary vector pVecBARII and phosphinothricin as a selective agent ${ }^{41} . \mathrm{T}_{0}$ plants were transplanted to a growth cabinet $\left(23^{\circ} \mathrm{C}, 16 \mathrm{~h} \mathrm{light} / 8 \mathrm{~h}\right.$ darkness) and inoculated with $P g t$ race 98-1,2,3,5,6+Sr50 at 7-10 days posttransplantation. Rust reactions were assessed $10-15$ days post $P g t$ inoculation ${ }^{28}$.

Rust phenotyping and histological assessment. Stem rust phenotyping of seedlings and adult plants in the greenhouse and field were as previously described ${ }^{42-44}$. Histological assessments of Pgt infection site sizes were as previously described by Ayliffe et al. ${ }^{4}$. Images were photographed using a CC12 digital camera and AnalySIS LS Research v2.2 software for analysis (Olympus Soft Imaging System, Japan).

Phylogenetic analysis. R gene protein sequences from the NCBI database (protein accession numbers are listed in Supplementary Data 1) were aligned using MUSCLE and phylogenetic trees were constructed using the UPGMA program in $\mathrm{MegaX}^{46}$. Evolutionary distances were determined using the Neighbour-Joining method ${ }^{47}$ with Poisson correction and units to show the number of amino acid substitutions per site. All positions that contained either gaps or missing data were removed from the analysis. Phylogenetic trees were annotated using ITOL (https:// itol.embl.de).

Protein structure predictions. Coiled-coil domains in predicted Sr26 and Sr61 proteins were indentified using the COILS prediction program ${ }^{48}$ (https://embnet. vital-it.ch/software/COILS_form.html) and the T-Coffee program Expresso (http:// tcoffee.crg.cat/apps/tcoffee/do:expresso) was used for protein sequence alignment.

Molecular cytogenetic characterization of lines containing Sr26 and Sr61. Root tips were pre-treated and slides were prepared according to Zhang et al. ${ }^{49}$. Nondenaturing fluorescence in situ hybridization (ND-FISH) with oligonucleotide probes OligopSc119.2-1 and Oligo-pTa535-1 was used to identify individual chromosomes ${ }^{50}$. OligopSc119.2-1 and Oligo-pTa535-1 were labeled with 6carboxyfluorescein (6-FAM) and 6-carboxytetramethylrhodamine (Tamra), generating green and red signals, respectively. Chromosomes were counterstained with 4',6-diamidino-2-phenylindole (DAPI) (Invitrogen) and pseudocolored blue. Photomicrographs were taken with a Retiga EXi CCD (charge-coupled device) camera (QImaging, Surrey, BC, Canada) mounted on a Zeiss Axio Imager epifluorescence microscope. After stripping off the oligo probes, the same slides were used to further characterize the Sr26 translocation line Avocet+Lr46, Sr61 substitution line W3757 and recombinant 378/15 by genomic in situ hybridization (GISH) as described by Zhang et al. ${ }^{49}$. Total genomic DNA from Pseudoroegneria stipifolia (accession PI 314058) was labeled with biotin-16-dUTP (Roche Diagnostics Australia, Castle Hill, NSW) using nick translation. Unlabeled total genomic DNA of wheat was used as blocker at a ratio of 1:80 (probe: blocker). Signals were detected with fluorescein avidin DN (Vector Laboratories, Burlingame, CA, USA). Chromosomes were counterstained with DAPI and pseudocolored red.

Characterization of T-DNA copy number by digital PCR. The phosphinothricin (PPT)-selectable marker gene located near the T-DNA left border was used to estimate the transgene copy number. The PPT primer pair and PPT-probe (labeled with 5'FAM (6-fluorescein) and doubled-quenched with ZENTM and Iowa Black Hole Quencher 1) were used according to Petrie et al. ${ }^{51}$. Genomic DNA (approximately $100 \mathrm{ng}$ ) was digested with EcoRI (New England Biolabs, Ipswich, MA, USA) in a final volume of $20 \mu \mathrm{L}$ for $4 \mathrm{~h}$ at $37^{\circ} \mathrm{C}$. Samples were placed onto Droplet Generator QX200TM (Bio-Rad) and heat sealed. Amplifications were carried in C1000 Thermal Cycler (Bio-Rad) and reactions were run with the following cycles: $95^{\circ} \mathrm{C}$ for $10 \mathrm{~min}$ followed by 40 cycles at $94^{\circ} \mathrm{C}$ for $30 \mathrm{~s} ; 59^{\circ} \mathrm{C}$ for 1 min, then $98^{\circ} \mathrm{C}$ for $10 \mathrm{~min}$ and maintained at $12^{\circ} \mathrm{C}$ finally. A ramping rate of $2.5^{\circ} \mathrm{C} / \mathrm{s}$ in all temperature change steps was employed. Following with amplification reaction, the plates were placed onto a QX200 Droplet Reader (Bio-Rad). Data was analysed using Quanta soft ${ }^{\mathrm{TM}}$ software (Bio-Rad).

QRT-PCR for determining transgene expression. Leaf tissues from each sample were frozen in liquid nitrogen or dry ice immediately after sampling. RNA was isolated using a RNeasy ${ }^{\circledast}$ Plant Mini Kit (QIAGEN, Chadstone Center, VIC, Australia) according to the manufacturer's protocol. One to two microgram of RNA samples were used for first-strand DNA synthesis in $20 \mu \mathrm{L}$ reactions using
Superscript ${ }^{\circledast}$ III reverse transcriptase kit (Life Technologies, Mulgrave, VIC, Australia). After the reverse transcript reaction, $3 \mu \mathrm{L}$ of $10 \times$ dilutions of synthesis product were used for $\mathrm{qPCR}$ reaction using a C1000 $\mathrm{Touch}^{\mathrm{TM}}$ thermocycler with the CFX96 ${ }^{\mathrm{TM}}$ Real-Time System (Bio-Rad). qPCR conditions included an initial denaturation at $95^{\circ} \mathrm{C}$ for $3 \mathrm{~min} ; 40$ cycles of denaturation at $95^{\circ} \mathrm{C}$ for $10 \mathrm{~s}$ and annealing/elongation at $60^{\circ} \mathrm{C}$ for $30 \mathrm{~s}$, followed by a melt step range of $65-95^{\circ} \mathrm{C}$ with an increment of $0.5^{\circ} \mathrm{C}$. We used the wheat housekeeping gene TaCON as a reference gene for each qRT-PCR experiment ${ }^{52}$. qPCR primers specific for $\mathrm{Sr} 61$ (Sr61GSPF1 and Sr61GSPR1) were used to measure relative gene expression (Supplementary Table 3). Experiments included three technical replicates for each of three biological replications. $\delta \mathrm{Cq}$ mean values were calculated and standard errors were determined. Gene expression values were log (base 2)-transformed.

Reporting summary. Further information on research design is available in the Nature Research Reporting Summary linked to this article.

\section{Data availability}

Data supporting the findings of this work are available within the paper and its Supplementary Information files. A reporting summary for this article is available as a Supplementary Information file. The datasets and plant materials generated and analyzed during the current study are available from the corresponding authors upon request. Annotated genomic sequences of Sr26 and Sr61 have been deposited at NCBI GenBank with accession numbers MN531843 (Sr26) and MN531844 (Sr61). The source data underlying Supplementary Figs. 3f, 4, and 7c are provided as a Source Data file. Source data are provided with this paper.

Received: 27 October 2019; Accepted: 10 May 2021;

Published online: 07 June 2021

\section{References}

1. Singh, R. P. et al. Emergence and spread of new races of wheat stem rust fungus: continued threat to food security and prospects of genetic control. Phytopathology 105, 872-884 (2015).

2. Li, F. et al. Emergence of the Ug99 lineage of the wheat stem rust pathogen through somatic hybridisation. Nat. Commun. 10, 5068 (2019).

3. Saintenac, C. et al. Identification of wheat gene Sr35 that confers resistance to Ug99 stem rust race group. Science 341, 783-786 (2013).

4. Mago, R. et al. The wheat $S r 50$ gene reveals rich diversity at a cereal disease resistance locus. Nat. Plants 1, 15186 (2015)

5. Zhang, W. et al. Identification and characterization of $\mathrm{Sr} 13$, a tetraploid wheat gene that confers resistance to the Ug99 stem rust race group. Proc. Natl Acad. Sci. USA 114, E9483-E9492 (2017).

6. Chen, S., Zhang, W., Bolus, S., Rouse, M. N. \& Dubcovsky, J. Identification and characterization of wheat stem rust resistance gene Sr21 effective against the Ug99 race group at high temperature. PLoS Genet. 14, e1007287 (2018).

7. Periyannan, S. et al. The gene Sr33, an ortholog of barley Mla genes, encodes resistance to wheat stem rust race Ug99. Science 341, 786-788 (2013).

8. Steuernagel, B. et al. Rapid cloning of disease-resistance genes in plants using mutagenesis and sequence capture. Nat. Biotechnol. 34, 652-655 (2016).

9. Arora, S. et al. Resistance gene cloning from a wild crop relative by sequence capture and association genetics. Nat. Biotechnol. 37, 139 (2019).

10. Chen, S. et al. Wheat gene Sr60 encodes a protein with two putative kinase domains that confers resistance to stem rust. New Phytol. 225, 948-959 (2020).

11. Knott, D. R. The inheritance of rust resistance. VI. The transfer of stem rust resistance from Agropyron elongatum to common wheat. Can. J. Plant Sci. 41, 109-123 (1961).

12. Dundas, I., Zhang, P., Verlin, D., Graner, A. \& Shepherd, K. Chromosome engineering and physical mapping of the Thinopyrum ponticum translocation in wheat carrying the rust resistance gene Sr26. Crop Sci. 55, 648-657 (2015).

13. McIntosh, R. A., Dyck, P. L. \& Green, G. J. Inheritance of leaf rust and stem rust resistances in wheat cultivars Agent and Agatha. Aust. J. Agric. Res. 28, 37-45 (1977)

14. Park, R. F. Stem rust of wheat in Australia. Aust. J. Agric. Res. 58, 558-566 (2007).

15. Zwer, P. K., Park, R. F. \& McIntosh, R. A. Wheat stem rust in Australia-19691985. Aust. J. Agric. Res. 43, 399-431 (1992).

16. Singh, R. P. \& McIntosh, R. A. Genetics of resistance to Puccinia graminis tritici in Chris and W3746 wheats. Theor. Appl. Genet. 73, 846-855 (1987).

17. Martin, R. H. Eagle-a new wheat variety. Agric. Gaz. N. South Wales 82, 206-207 (1971)

18. Jenkin, M., Shepherd, K. W. \& Brown, A. H. D. Isozyme variation associated with the 9A-1 Agropyron translocation in wheat and genetic mapping of its breakpoint. Waite Agricultural Research Institute Biennial Report (1984). 
19. Mago, R. et al. Transfer of stem rust resistance gene $\mathrm{SrB}$ from Thinopyrum ponticum into wheat and development of a closely linked PCR-based marker. Theor. Appl. Genet. https://doi.org/10.1007/s00122-018-3224-1 (2019).

20. Mago, R. et al. Development of PCR markers for the selection of wheat stem rust resistance genes Sr24 and Sr26 in diverse wheat germplasm. Theor. Appl. Genet. 111, 496-504 (2005).

21. Liu, S. et al. Diagnostic and co-dominant PCR markers for wheat stem rust resistance genes Sr25 and Sr26. Theor. Appl. Genet. 120, 691-Sr697 (2010).

22. Zhang, J. et al. A strategy for identifying markers linked with stem rust resistance in wheat harbouring an alien chromosome introgression from a non-sequenced genome. Theor. Appl. Genet. https://doi.org/10.1007/s00122018-3201-8 (2018).

23. Zhang, J., Zhang, P., Dodds, P. \& Lagudah, E. How target-sequence enrichment and sequencing (TEnSeq) pipelines have catalyzed resistance gene cloning in the wheat-rust pathosystem. Front. Plant Sci. 11, 678 (2020).

24. Hatta, M. A. M. et al. The wheat $S r 22, S r 33, S r 35$ and $S r 45$ genes confer resistance against stem rust in barley. Plant Biotechnol. J. https://doi.org/ 10.1111/pbi.13460 (2020)

25. Wang, H. et al. Horizontal gene transfer of $F h b 7$ from fungus underlies Fusarium head blight resistance in wheat. Science https://doi.org/10.1126/ science.aba5435 (2020).

26. Kourelis, J. \& van der Hoorn, R. A. L. Defended to the nines: 25 years of resistance gene cloning identifies nine mechanisms for $\mathrm{R}$ protein function. Plant Cell 30, 285-299 (2018).

27. Cui, L. et al. Development of perennial wheat through hybridization between wheat and wheatgrasses: a review. Engineering 4, 507-513 (2018).

28. McIntosh, R. A., Wellings, C. R. \& Park, R. F. Wheat Rusts: An Atlas of Resistance Genes (CSIRO, 1995).

29. Mago, R. et al. Development of wheat-Aegilops speltoides recombinants and simple PCR-based markers for Sr32 and a new stem rust resistance gene on the 2S\#1 chromosome. Theor. Appl. Genet. 126, 2943-2955 (2013).

30. Luo, M. et al. A five-transgene cassette confers broad-spectrum resistance to a fungal rust pathogen in wheat. Nat. Biotechnol. https://doi.org/10.1038/ s41587-020-00770-x (2021)

31. Klymiuk, V. et al. Cloning of the wheat Yr15 resistance gene sheds light on the plant tandem kinase-pseudokinase family. Nat. Commun. https:/doi.org/ 10.1038/s41467-018-06138-9 (2018).

32. Marchal, C. et al. BED-domain-containing immune receptors confer diverse resistance spectra to yellow rust. Nat. Plants 4, 662-668 (2018).

33. Wang, H., Zou, S. H., Li, Y. W., Lin, F. Y. \& Tang, D. Z. An ankyrin-repeat and WRKY-domain-containing immune receptor confers stripe rust resistance in wheat. Nat. Commun. https://doi.org/10.1038/s41467-020-15139-6 (2020).

34. Zhang, C. et al. An ancestral NB-LRR with duplicated 3'UTRs confers stripe rust resistance in wheat and barley. Nat. Commun. 10, 4023 (2019).

35. Cloutier, S. et al. Leaf rust resistance gene $\operatorname{Lr} 1$, isolated from bread wheat (Triticum aestivum L.) is a member of the large psr567 gene family. Plant Mol. Biol. 65, 93-106 (2007).

36. Huang, L. et al. Map-based cloning of leaf rust resistance gene $L r 21$ from the large and polyploid genome of bread wheat. Genetics 164, 655-664 (2003).

37. Feuillet, C. et al. Map-based isolation of the leaf rust disease resistance gene Lr10 from the hexaploid wheat (Triticum aestivum L.) genome. Proc. Natl Acad. Sci. USA 100, 15253-15258 (2003).

38. Kolodziej, M. C. et al. A membrane-bound ankyrin repeat protein confers racespecific leaf rust disease resistance in wheat. Nat. Commun. 12, 956 (2021).

39. Wulff, B. B. H. \& Dhugga, K. S. Wheat-the cereal abandoned by GM. Science 361, 451-452 (2018).

40. Yu, G., Hatta, A., Periyannan, S., Lagudah, E. \& Wulff, B. B. H. Isolation of wheat genomic DNA for gene mapping and cloning. Methods Mol. Biol. 1659, 207-213 (2017).

41. Ishida, Y., Tsunashima, M., Hiei, Y. \& Komari, T. Wheat (Triticum aestivum L.) transformation using immature embryos. Agrobacterium Protoc. 1223, 189-198 (2015).

42. Pretorius, Z. A. et al. Assessing the vulnerability of wheat germplasm from Bangladesh and Nepal to Ug99 stem rust. Phytoparasitica 43, 637-645 (2015).

43. Bender, C. M., Prins, R. \& Pretorius, Z. A. Development of a greenhouse screening method for adult plant response in wheat to stem rust. Plant Dis. 100, 1627-1633 (2016).

44. Stakman, E. C., Stewart, D. M. \& Loegering, W. Q. Identification of physiological races of Pucinia graminis var. tritici. USDA Research Service Bulletin (1962).

45. Ayliffe, M. et al. A simple method for comparing fungal biomass in infected plant tissues. Mol. Plant Microbe Interact. 26, 658-667 (2013).

46. Kumar, S., Stecher, G., Li, M., Knyaz, C. \& Tamura, K. MEGA X: molecular evolutionary genetics analysis across computing platforms. Mol. Biol. Evol. 35, 1547-1549 (2018).
47. Saitou, N. \& Nei, M. The neighbor-joining method: a new method for reconstructing phylogenetic trees. Mol. Biol. Evol. 4, 406-425 (1987).

48. Lupas, A., Van Dyke, M. \& Stock, J. Predicting coiled coils from protein sequences. Science 252, 1162-1164 (1991).

49. Zhang, P., Friebe, B., Lukaszewski, A. J. \& Gill, B. S. The centromere structure in Robertsonian wheat-rye translocation chromosomes indicates that centric breakage-fusion can occur at different positions within the primary constriction. Chromosoma 110, 335-344 (2001).

50. Tang, Z., Yang, Z. \& Fu, S. Oligonucleotides replacing the roles of repetitive sequences pAs1, pSc119.2, pTa-535, pTa71, CCS1, and pAWRC.1 for FISH analysis. J. Appl. Genet. 55, 313-318 (2014)

51. Petrie, J. R. et al. Development of a Brassica napus (Canola) crop containing fish oil-like levels of DHA in the seed oil. Front. Plant Sci. 11, 727 (2020).

52. Moore, J. W. et al. A recently evolved hexose transporter variant confers resistance to multiple pathogens in wheat. Nat. Genet. 47, 1494-1498 (2015).

\section{Acknowledgements}

This work was supported by National Science Foundation, Basic Research Enabling Agriculture Development (NSF-BREAD), USA, and the Grains Research and Development Corporation (GRDC), Australia. The first author was supported by the Monsanto Beachell-Borlaug International Scholars Programs (MBBISP), USA, and the Research Training Program (RTP) of the Australian Department of Education and Training.

\section{Author contributions}

J.Z. performed all the experiments to clone $S r 26$ and $S r 61$ and analysed the data. Sr26 and Sr61 mutants were generated by P.Z., R.A.M., S.H., W.S., and E.L. Rust pathology work in South Africa, Denmark, and Australia was conducted by W.H.B., Z.A.P., M.P., M.H., P. Z., R.A.M., J.Z., and R.F.P. NGS data analysis for candidate gene identification of $\mathrm{Sr} 26$ was by T.C.H., N.U., SP., and J.Z., and for the Sr61 candidate was carried out by J.Z. Design and testing of gene-specific markers was by J.Z., S.C., and E.L. J.Z., D.B., S.C., and M.L. performed the transgenic validation of $S r 26$ and Sr61 with oversight by M.A. Molecular cytogenetic experiments to characterize the wild-type and recombinant lines combining Sr26 and Sr61 were carried out by J.L. and P.Z. Phylogenetic tree was constructed and analysed by J.Z. and P.D. Initial development and characterization of recombinants were done by I.D. NLR bait libraries were developed and provided by B.B. H.W. and B.S. E.L., P.Z., and R.A.M. conceived, designed, and supervised the research. J. Z., E.L., R.A.M., P.Z., and M.A. drafted the manuscript and all co-authors provided edits

\section{Competing interests}

Patent applications based on this work have been filed by E.L., P.Z., and J.Z. and the filing reference numbers for Sr26 and Sr61 are PCT/AU2019/050331 and PCT/AU2020/ 051224 , respectively. Other authors claim no competing interests.

\section{Additional information}

Supplementary information The online version contains supplementary material available at https://doi.org/10.1038/s41467-021-23738-0.

Correspondence and requests for materials should be addressed to P.Z. or E.S.L.

Peer review information Nature Communications thanks Colin Hiebert, Yue Jin, and other anonymous reviewers for their contributions to the peer review of this work. Peer review reports are available.

Reprints and permission information is available at http://www.nature.com/reprints

Publisher's note Springer Nature remains neutral with regard to jurisdictional claims in published maps and institutional affiliations.

Open Access This article is licensed under a Creative Commons Attribution 4.0 International License, which permits use, sharing, adaptation, distribution and reproduction in any medium or format, as long as you give appropriate credit to the original author(s) and the source, provide a link to the Creative Commons license, and indicate if changes were made. The images or other third party material in this article are included in the article's Creative Commons license, unless indicated otherwise in a credit line to the material. If material is not included in the article's Creative Commons license and your intended use is not permitted by statutory regulation or exceeds the permitted use, you will need to obtain permission directly from the copyright holder. To view a copy of this license, visit http://creativecommons.org/licenses/by/4.0/. 